\title{
Designing Information Systems Requirements in Context: Insights from the Theory of Deferred Action
}

\author{
Nandish V. Patel and Ray Hackney \\ Information Systems Evaluation and Integration Network Group (ISEing) \\ Brunel Business School \\ Brunel University, UK
}

\begin{abstract}
This paper considers conceptual and contextual issues relating to the problem of developing systems models capable of representing knowable and unknowable information requirements. It postulates that the systemic emergence property of systems is the cause of unknowable organisational information requirements. The Theory of Deferred Action and its systems constructs are invoked to explain emergence and how it can be modelled in formal systems. The insights proposed are believed to assist designers in developing functional and relevant approaches within dynamic organisational contexts.
\end{abstract}

Keywords: complexity, information requirements, information systems, modelling, systems design, systemic emergence, theory of deferred action, deferred systems

\section{INTRODUCTION}

Emergence affects actors' information requirements. The assumption that information requirements are stable is negated under emergent conditions. Events that cannot be predicted, and therefore cannot be planned, are emergent events. 'Emergence is an unpredictable effect of the interrelatedness of multiple purposes and the multifarious means to achieve them that is characteristic of social action. By implication, emergence is a nonspecifiable constraint on rational design because it cannot be clearly defined beforehand (Patel, 2006:12). Emergence is a core feature of complexity science, which views complex systems as a constant 'phase change' arising from emergence. Emergence requires adaptation, resulting in 'complex adaptive systems' (McMillan, 2004). These systems are often selforganizing in nature. Complexity in turn requires appropriate concepts and constructs of knowledge. The ability to spontaneously adapt and self-organisation are aspects of organisations that are not generally considered in theories of knowledge management. This paper considers theoretically the effect of systemic emergence on information requirements and discusses techniques suitable for modelling emergence in information systems.

There is evidence to suggest that designers have not adequately addressed many of the organisational problems relating to the appropriate functionality of information systems (IS) (Jürgen, 2005; Patel, 2005). In terms of designing IS how should a contemporary organization and the design of formal systems relate? The concerns relate to the actual context under consideration, which is often unpredictable and boundless, with formal systems design, which is theoretically predictable and finite. It is a problem of designing systems for 'actuality', i.e. for applied sustainable business situations (Hackney et al, 2007). Historically, practitioners have engaged with various pragmatic approaches to systems design (Dasgupta, 1989). For example, structured, object oriented and agile systems analysis. Recently however, new 
processes appear to recognize more directly the unpredictable and informal nature of the actuality for which formal designs are created. Consequently, successive new IS design approaches relegate formalism and promote, currently and most radically, agile systems development. The aim of this paper is to consider the basis of these design approaches which may enable useful insights into the critical information needs of an organisation relative to the formal design of IS.

The concepts within the Systems Approach is central to develop understanding of how rationally designed formal systems behave in context. Different conceptions have evolved as new challenges have been met since the emergence of General Systems Theory (GST) (1968) and Boulding, (1956). These include the Turing Machine (Turing, 1950), system dynamics (Forrester, 1961), socio-technical systems (Keller, 1996) hard and soft systems thinking (Checkland, 1972). They are all different representations of formal systems proposed to address the actuality in which designed systems are required to operate. Successive approaches have sought various compilations of empirical data on human intelligence, representation of organizational facts, organizational change, and human interpretation that need to be reflected in systems design. The Theory of Deferred Action is proposed as an explanation of how formally designed systems relate with actuality. Whilst other approaches within systems development give less importance to actuality the deferred action theoretic merges the actual with the formal (Patel, 2006). It usefully affirms the importance of system conceptions within IS design and also integrates the actual as part of the formal design. The deferred action theoretic is therefore considered valuable in exploring the development of systems analysis and design techniques necessary for organization effectiveness.

\section{CONTEXTUAL APPROACHES}

Systems are frequently assumed to be essentially static and systems analysis and design techniques are devised to model 'stable data' (Fitzgerald, 1990; Truex et al, 199). This notion is the basis for ANSI-SPARC schemas for database design (Tsichritzis and Klug, 1975) and data (Tsichritzis and Lochosky, 1982). They address only structure and knowable functional requirements as design based on a given specification, usually derived through initial systems analysis of the problem that is the derived specification as depicted in Figure 1.

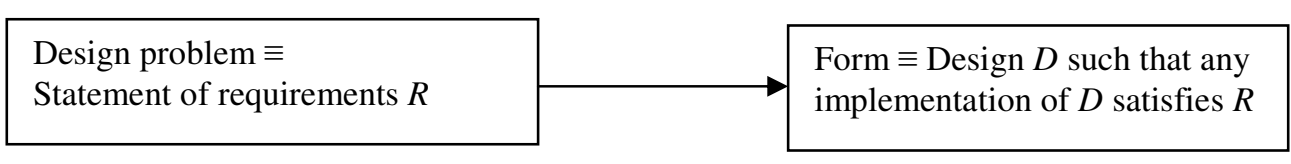

Figure 1 Model of design process as specification design

Source: (Dasgupta, 1989)

Dasgupta's (1989) suggests a design problem is essentially some statement of requirements $R$ that designers then convert into some solution design $\mathrm{D}$ that satisfies $\mathrm{R}$. Requirements are assumed not only to be static but also knowable in this model of the design process.

In addition, systems analysis techniques that recognize organizational change such as flexibility analysis (Fitzgerald, 1991) and futures analysis (Land, 1982) assume the nature of the system to be static which may be represented through a formal specification of information requirements. The subfield of dynamical systems (Jürgen, 2005) similarly subscribes to this model. Futures analysis and dynamical systems are also based on the ability of systems analysts to make predictions, a highly dubious proposition. Whilst the futures analysis technique and systems dynamics depend on analysts' power of prediction for success, the flexibility analysis technique does not. It proposes the setting up of a high level 
flexibility analysis team capable of investigating: "potential changes to the organization, the business, and the environment in which the system is being developed in the short, medium and long term.' (Fitzgerald, 1991: 9).

\section{THEORETICAL ANALYSIS}

We may usefully categorise the information needs of an organization into knowable information requirements Rk and unknowable information requirements Ŕ $\mathrm{u}$, as depicted in Figure 2. This is believed to represent an accurate description of the context in which formally designed organization and systems have to function. By implication, the design of a formal system would need models and algorithms that provide some kind of correspondence with both kinds of requirements. This correspondence cannot be a one-to-one correspondence because of the criterion of unknowability of information requirements. Formal systems design though can only cope with the knowable requirements R. This is because the basis of IS represents commonly an algorithmic procedure based on specified requirements which are finite, bounded and rational.

\begin{tabular}{|l|l|}
$\begin{array}{l}\text { Design problem } \equiv \text { knowable } \\
\text { requirements } \mathrm{Rk} \text { and unknowable } \\
\text { requirements Ru }\end{array}$ & $\begin{array}{l}\text { Form } \equiv \text { Design } D \text { such that any } \\
\text { implementation of } D \text { satisfies } R k \text { but } \\
\text { not } R u\end{array}$ \\
\hline
\end{tabular}

Figure 2 Insufficient Design Process

This design process is insufficient as it cannot cope with the unknowable requirements through the systems specification. Specification in turn stems from basing design on a conception of systems as formal systems. The formal systems conception itself is not totally invalid for the design of knowable and unknowable information requirements. Systems analysis tools are, as noted, developed mainly for knowable and specifiable information requirements. However, the designed specified systems have to function in an organization with unknowable information requirements, here termed emergence.

The characterisation of information requirements as knowable and unknowable is theoretically plausible given the substantive nature of systems. Systems theory asserts that emergence, hierarchy, communications and control (EHCC) are related properties (Checkland, 1972). The idea of emergence is central in GST (Churchman, 1968) and systems thinking (Checkland, 1972). Of these properties emergence and hierarchy lend support to thinking of information as knowable and unknowable. Emergence is the theoretical consequence of the unknowable information requirements. But there appears to be a limited systems analysis conception or techniques for emergence in contemporary design.

\section{EMERGENCE PROPOSITION}

To assume that emergence can be predicted is logically flawed. Systems analysis and systems design based on predicting the shape of systems, where shape equals its structure (architecture) and operations, is incorrect. Systems thinking and systems dynamics, and other systems analysis techniques assume implicitly that emergence can be predicted. This is because, as noted, they assume the system to be static. Soft Systems Methodology (Checkland, 1972) also implicitly recognizes emergence, but it is flawed because the very act of modelling it assumes it can be predicted. 
There is no research on systems and emergence and emergent systems in systems analysis and systems design. Emergent systems cater for the property of emergence that GST and Systems Thinking attribute to systems. Emergence itself is the result of the hierarchy property of systems. However, emergence as a property is taken by extent systems analysis and design techniques like SSM and systems dynamics to result in predictable or a specifiable system.

There is no explicit recognition of emergence in systems analysis and design and there is no design for it in systems design. Researches have not sought to develop theoretical understanding of the implications of emergence for systems analysis and design and techniques for emergence analysis have not been proposed. In object oriented analysis for example, there are no techniques to identify emergent objects or emergence of the system in general. Practitioners following agile systems development have developed techniques to cope with emergent information needs but the underlying conception of systems is that they can be specified or predicted.

Recognition of emergence in GST and Systems Thinking is significant, but it is not framed in a way that is useful for practical IS systems analysis and design. Given emergent systems, what kind of systems analysis and design is required? This question is addressed theoretically in the next section. It is necessary to understand and develop theoretical knowledge for emergence management during systems analysis and design and how it can be represented in the design of systems for organizations. The practical implications for designing techniques to model emergent systems are discussed in the penultimate section. This is done by drawing on the tailoring facility of UML to adapt its notation to model emergence.

\section{THEORY OF DEFERRED ACTION}

Theoretical and actual emergence is addressed in the Theory of Deferred Action (Patel, 2006). The theory proposes that systems should be analysed along four dimensions. These dimensions are planned action (or rationally designed formal systems), emergence (things that cannot be known for design purposes), deferred action (enabling natural design, the things humans do naturally to achieve purpose, within rational design) and diffused management. Emergence in actuality is a central tenant of the theory. Actual emergence cannot be predicted and therefore cannot be the basis for systems design. Thinking of systems with these four dimensions gives four types of systems design: deferred systems, real systems, specified systems and autonomous systems, depicted in Figure 3.

The focus in this paper is on deferred systems (point A) and the systems analysis and design of deferred systems. Deferred systems cater for unknowable information requirements. They are emergent systems in which the operations (functionality) of the system emerge during execution. (In real systems, point A, both the structure or architecture and operations emerge during execution. For further details of such real systems see Patel, 2006). The structure and operations of an emergent system cannot be planned or predefined. Structure and operations cannot be predicted. Therefore they need to be deferred. 


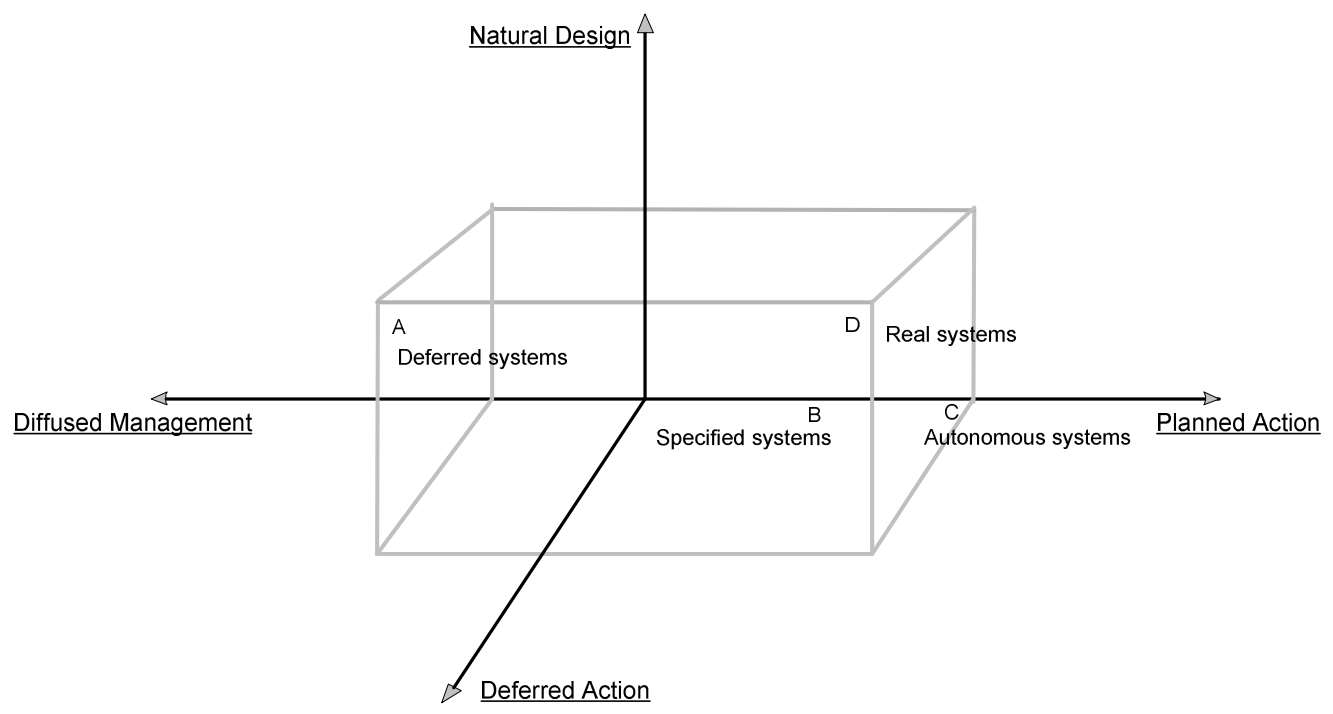

Figure 3 Space of systems design

Source: Patel (2006)

An analysis of systems analysis techniques in terms of the theory of deferred action classification of systems shown in Figure 3 reveals that research is focused on specified systems and much of its ontological commitment is positivist or implicitly positivist. Deferred systems and real systems research utilizes the design science epistemology. Where no epistemology is explicitly stated the research may be described as pragmatist. Techniques that focus on the present situation aim to establish the functionality presently required and they are mostly based on GST (Boulding, 1956) and the Systems Approach (Churchman, 1968). Techniques that recognise organizational change aim to establish what functionality is required in the future (Land, 1982; Fitzgerald, 1990). These techniques can be subdivided into predictive techniques (Land, 1982) and probabilistic techniques (Fitzgerald, 1990). All these techniques are termed discrete systems analysis techniques. Continuous systems analysis techniques are less evident, they occur in agile systems development. The techniques based on the theory of deferred action aim to establish what structural and functional design can be deferred. This is termed deferral analysis or deferment point analysis (Patel, 2006).

The aim of deferred systems research is to create systems analysis and design techniques for emergence analysis and to create design sensitive to emergence. It seeks a form of continuous systems analysis, as described in the final column of Table 1. Whereas specified systems focuses on the present and stable information requirements or discrete systems analysis, deferred systems analysis proposes the invention of technique for continuous systems analysis and conceptualises systems as continuous and emergent.

The emergence property of systems in GST and systems thinking is central in the deferred action theory as one of the four dimensions descriptive of systems. These accounts of emergence resonate with the deferred action theory. In the theory emergence necessitates deferred action necessary in reality to make formally designed systems relevant to actuality. Such emergence is a significant aspect of reality that affects systems design. The deferred action dimension is the design response necessary to allow systems to function in emergent spaces.

There is theoretical discussion and empirical evidence of emergent organization. The theoretical emergence in systems thinking and GST is supported with empirical evidence. 
Descriptions of organisations as emergent in terms of knowledge (Markus, 2002; Snowdon, 2003) and information (Truex, 1999; Patel 1999; Patel 2005) and information needs emerging in the course of some other activity (Sweeny, 1996) necessitate invention of systems analysis techniques and to include in systems design as practical mechanisms. Emergence ranges from strategic matters to technical and operational issues.

\section{SYSTEMS ANALYSIS AND SESIGN TECHNIQUES}

Systems analysis techniques for deferred systems design differ from those used in specified systems design. Based on the proposition that deferred action is necessary to enable systems to cope with emergence, systems analysis techniques have been proposed (Patel, 2005). These techniques aim to identify organization deferment points (ODPs), which are essentially emergent situations for which the organization (or individual or group) require a response. The situation may be new for which no formal response exists or it may occur within an existing formal systems design. Such ODPs need to be converted into systemic deferment points (SDPs) to make systems responsive to emergence. A SDP is a mechanism within a formal system design capable of enabling continuous systems design or deferred systems design.

The Information Systems Evaluation and Integration Centre (ISEing) at Brunel Business School is exploring UML extensions to model SDPs. The UML encourages tailoring of its notation for specific modelling needs. In this sense the UML is a deferred system because it permits it shape to be determined locally. The UML notation being considered that is the subject of a forthcoming paper is use case and use case extension points. A use case extension is shown in Figure 4.

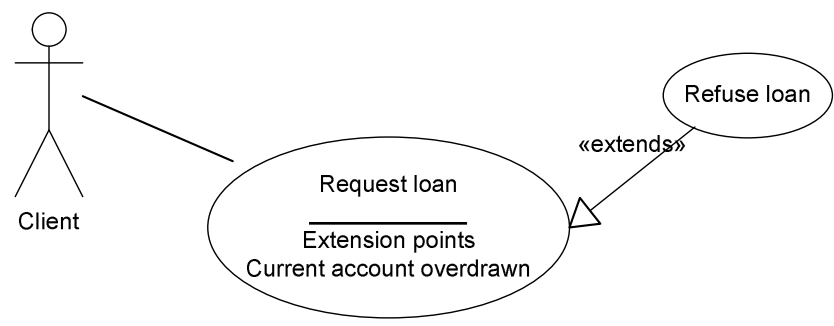

Figure 4 Use Case Extension Points

Figure 4 shows a use case for a loan request. It contains an extension point showing refuse loan because the current account is overdrawn. This facility can be extended to model SDPs and current work is exploring the kind of notation that would be required to depict such SDPs.

\section{CONCLUSION}

This paper identifies emergence from GST and systems thinking as a critical feature of systems analysis and design. It notes that there is little theoretical understanding of emergence in systems analysis and design and no practical techniques to model emergence. It proposes the four dimensional analysis based on the Theory of Deferred Action and its resultant constructs, particularly deferred systems, to enable systems analysis of emergence and its modeling for systems design. Deferred action is a synthesis of rationalism as has been applied to systems analysis and design and new research that identifies emergent organization as significant. Present research adopts the design science research method and it is focusing on extending the UML to devise notation capable of modeling knowable and unknowable information requirements in terms of formal systems design as deferred systems. Since design science is concerned with the utility of a designed artifact, additional research is needed to 
apply the deferred action constructs as a design science research effort. This will contribute to improving understanding of systems analysis and design and its utility in practice. 


\section{References}

Boulding K (1956), General Systems Theory - The Skeleton of Science. Management Science, April 1956: 197-208

Checkland, P. (1972). Towards a systems-based methodology for real-world problem-solving. Journal of Applied Systems Engineering, 3(2): 87-116.

Churchman C W (1968) Challenge to reason. McGraw-Hill. New York.

Churchman C W (1971) The Design of inquiring systems: basic concepts of systems and organization. Basic Books, New York.

Dasgupta S (1989) Computer architecture: a modern synthesis Volume 1, Foundations. Wiley, Chichester.

Forrester J W (1961) Industrial Dynamics. Cambridge MA: MIT Press. Reprinted by Productivity Press, Portland, Oregon.

Hackney R A, Jones S and Losch A, (2007) eReverse Auctions: towards an eGovernment framework European Journal Information Systems, Vol 16, pp178-191

Jürgen J (2005) Dynamical systems: examples of complex behaviour. Springer. Berlin.

Keller K (1996) Socio-technical systems and self-organization. SIGIOS Bulletin, 17 (1) April 1996.

Markus L M, Majchrzak A and Gasser L (2002) A Design Theory for Systems that Support Emergent Knowledge Processes. MIS Quarterly, 26 2002:179-212.

Patel N V (1999) The Spiral of Change Model for Coping with Changing and ongoing Requirements. Requirements Engineering. 4:77-84

Patel N V (2005) Sustainable systems: strengthening knowledge management systems with deferred action. Int. J. Information Technology and Management, 4 (4) 344-365.

Patel N V (2006) Organization and systems design: Theory of deferred action. Palgrave Macmillan, London.

Truex D P, Baskerville R and Klein H K (1999) Growing systems in an emergent organization. Communications of the ACM, 42 (8): 117-123.

Tsichritzis D and Klug A (1975) The ANSI spark report on the study group on database management systems. Information Systems, vol. 3

Tsichritzis D and Lochovsky F (1982) Data Models. Prentice Hall, Englewood, Cliff. NJ.

Turing A M (1950) Computing machinery and intelligence. Mind, 59 (236)

McMillan, E. (2004) Complexity, Organizations and Change. Routledge, London. 